\title{
Correction to: Blockchain and Crypto Currency
}

Makoto Yano, Chris Dai, Kenichi Masuda and Yoshio Kishimoto

Correction to:

M. Yano et al. (eds.), Blockchain and Crypto Currency, Economics, Law, and Institutions in Asia Pacific, https://doi.org/10.1007/978-981-15-3376-1

The original version of the book was inadvertently published with incorrect book title and coeditors' affiliations in the front matter. The corrected book has been updated with the changes. 